\title{
Legendre Polynomials Operational Matrix Method for Solving Fractional Partial Differential Equations with Variable Coefficients
}

\author{
Yongqiang Yang, Yunpeng Ma, and Lifeng Wang \\ School of Aeronautic Science and Technology, Beihang University, Beijing 100191, China \\ Correspondence should be addressed to Yunpeng Ma; mayunpeng088@163.com
}

Received 27 January 2015; Accepted 4 May 2015

Academic Editor: Francesco Pesavento

Copyright (C) 2015 Yongqiang Yang et al. This is an open access article distributed under the Creative Commons Attribution License, which permits unrestricted use, distribution, and reproduction in any medium, provided the original work is properly cited.

\begin{abstract}
A numerical method for solving a class of fractional partial differential equations with variable coefficients based on Legendre polynomials is proposed. A fractional order operational matrix of Legendre polynomials is also derived. The initial equations are transformed into the products of several matrixes by using the operational matrix. A system of linear equations is obtained by dispersing the coefficients and the products of matrixes. Only a small number of Legendre polynomials are needed to acquire a satisfactory result. Results obtained using the scheme presented here show that the numerical method is very effective and convenient for solving fractional partial differential equations with variable coefficients.
\end{abstract}

\section{Introduction}

The subject of factional calculus was found over 300 years ago. The theory of integrals and derivatives of noninteger order goes back to Leibnitz, Liouville, and Letnikov. In recent years, fractional derivative and fractional differential equations have played a very significant role in many areas in fluid flow, physics, mechanics, and other applications. A lot of practical problems can be elegantly modeled with the help of the fractional derivative [1-5]. Fractional derivatives provide an excellent instrument for the description of memory and hereditary properties of various materials and processes. Due to the increasing applications, a lot of attention has been paid to numerical and exact solution of fractional differential equations and fractional partial equations. The analytical solutions of fractional differential equations are still in a preliminary stage. Except in a limited number of these equations, we have difficulty in seeking their analytical as well as numerical solutions. Thus there have been attempts to develop the methods for getting analytical and numerical solutions of fractional differential equations. Recently, some methods have drawn attention, such as Adomian decomposition method (ADM) $[6,7]$, variational iteration method (VIM) [8], generalized differential transform method (GDTM) [9-11], finite difference method (FDM) [12], and wavelet method [13, 14].

In this paper, our study focuses on a class of fractional partial differential equations as follows:

$$
a(x) \frac{\partial^{\alpha} u(x, t)}{\partial x^{\alpha}}+b(x) \frac{\partial^{\beta} u(x, t)}{\partial t^{\beta}}=f(x, t),
$$

such that the initial conditions

$$
\begin{gathered}
u(x, 0)=g(x), \\
u(0, t)=h(t),
\end{gathered}
$$

where $\partial^{\alpha} u(x, t) / \partial x^{\alpha}$ and $\partial^{\beta} u(x, t) / \partial t^{\beta}$ are fractional derivatives of Caputo sense, $0<\alpha, \beta \leq 1, f(x, t), a(x)$, and $b(x)$ are the known and $u(x, t)$ is the unknown.

There have been several methods for solving the fractional partial differential equation. Doha et al. used Jacobi tau approximation to solve the numerical solution of the space fractional diffusion equation [15]. Yi et al. [16] applied block pulse functions method to obtain the fractional partial equations. Podlubny [17] obtained the numerical solution of the fractional partial differential equations with constant coefficients by using Laplace transform method. 


\section{Definitions of Fractional Derivatives and Integrals}

Definition 1. Riemann-Liouville fractional integral of order $\alpha,(\alpha \geq 0)$ is defined as follows [17]:

$$
\begin{aligned}
& I^{\alpha} u(t)=\frac{1}{\Gamma(\alpha)} \int_{0}^{t}(t-\tau)^{\alpha-1} u(\tau) d \tau, \quad t>0, \\
& I^{0} u(t)=u(t)
\end{aligned}
$$

where $\Gamma(\alpha)=\int_{0}^{\infty} t^{\alpha-1} e^{-t} d t$ is the gamma function.

The Riemann-Liouville fractional integral satisfies the following properties:

$$
\begin{aligned}
I^{\alpha} I^{\beta} u(t) & =I^{\beta} I^{\alpha} u(t), \\
I^{\alpha} I^{\beta} u(t) & =I^{\alpha+\beta} u(t), \\
I^{\alpha} t^{r} & =\frac{\Gamma(r+1)}{\Gamma(\alpha+r+1)} t^{\alpha+r} .
\end{aligned}
$$

Definition 2. Caputo's fractional derivative of order $\alpha,(\alpha \geq 0)$ is defined as follows [17]:

$$
\begin{aligned}
D_{*}^{\alpha} u(t)=\frac{1}{\Gamma(r-\alpha)} \int_{0}^{t} \frac{u^{r}(\tau)}{(t-\tau)^{\alpha-r+1}} d \tau, & \\
& 0 \leq r-1<\alpha<r .
\end{aligned}
$$

Particularly, the operator $D_{*}^{\alpha}$ satisfies the following properties ( $c$ is a constant):

$$
\begin{aligned}
& D_{*}^{\alpha} c=0, \\
& D_{*}^{\alpha} t^{\beta} \\
& = \begin{cases}0, & \beta \in \mathbf{N}_{0}, \quad \beta<\lceil\alpha\rceil, \\
\frac{\Gamma(\beta+1)}{\Gamma(\beta+1-\alpha)} t^{\beta-\alpha}, & \beta \in \mathbf{N}_{0}, \quad \beta \geq\lceil\alpha\rceil ; \beta \notin \mathbf{N}, \beta>\lfloor\alpha\rfloor,\end{cases} \\
& D_{*}^{\alpha} I^{\alpha} u(t)=u(t), \\
& I^{\alpha} D_{*}^{\alpha} u(t)=u(t)-\sum_{k=0}^{r-1} u^{(k)}\left(0^{+}\right) \frac{t^{k}}{k !}, \quad t>0, \\
& D_{*}^{\alpha}(\lambda f(t)+\mu g(t))=\lambda D_{*}^{\alpha}(f(t))+\mu D_{*}^{\alpha}(g(t)) .
\end{aligned}
$$

\section{Legendre Polynomials and Some of Their Properties}

The Legendre basis polynomials of degree $n$ in $[0,1]$ (see [18]) are defined by

$$
P_{i+1}(t)=\frac{(2 i+1)(2 t-1)}{(i+1)} P_{i}(t)-\frac{i}{i-1} P_{i-1}(t),
$$

where $P_{0}(t)=1, P_{1}(t)=2 t-1$. The Legendre polynomials of degree $i$ can be also written as

$$
P_{i}(t)=\sum_{k=0}^{i}(-1)^{i+k} \frac{(i+k) !}{(i-k) !} \frac{t^{k}}{(k !)^{2}} .
$$

Let

$$
\Phi(t)=\left[P_{0}(t), P_{1}(t), \ldots, P_{n}(t)\right]^{T}
$$

The Legendre polynomials given by (7) can be expressed in the matrix form

$$
\Phi(t)=\mathbf{A T}_{n}(t)
$$

where

$$
\begin{aligned}
& \mathbf{A} \\
& =\left[\begin{array}{ccccc}
1 & 0 & 0 & \cdots & 0 \\
-1 & (-1)^{2} 2 ! & 0 & \cdots & 0 \\
(-1)^{2} & (-1)^{3} \frac{3 !}{1 !} & (-1)^{4} \frac{4 !}{2 !} & \cdots & 0 \\
\vdots & \vdots & \vdots & \ddots & \vdots \\
(-1)^{n} & (-1)^{n+1} \frac{(n+1) !}{(n-1) !} & (-1)^{n+1} \frac{(n+2) !}{(n-2) ! 2 !} & \cdots & (-1)^{2 n} \frac{(2 n) !}{n !}
\end{array}\right], \\
& \mathbf{T}_{n}(t)=\left[1, t, \ldots, t^{n}\right]^{T} .
\end{aligned}
$$

Obviously

$$
\mathbf{T}_{n}(t)=\mathbf{A}^{-1} \boldsymbol{\Phi}(t)
$$

A function $u(t) \in L^{2}(0,1)$ can be expressed in terms of the Legendre basis. In practice, only the first $(n+1)$ term of Legendre polynomials is considered. Hence

$$
u(t) \cong \sum_{i=0}^{n} c_{i} P_{i}(t)=\mathrm{c}^{T} \Phi(t)
$$

where $\mathbf{c}=\left[c_{0}, c_{1}, \ldots, c_{n}\right]^{T}, c_{i}(i=0,1,2, \ldots, n)$ are called Legendre coefficients.

We extend the notion to two-dimensional space and define two-dimensional Legendre polynomials of order $\widehat{m}=$ $n+1$ as a product function of two Legendre polynomials:

$$
\begin{aligned}
& P_{\Pi}(x, t)=P_{a}(x) P_{b}(t), \\
& \Pi=\widehat{m} a+b+1, a, b=0,1,2, \ldots, n .
\end{aligned}
$$

For the function $u(x, t) \in L^{2}([0,1] \times[0,1])$, we can also get its approximation by using Legendre polynomials:

$$
u(x, t) \cong \sum_{i=0}^{n} \sum_{j=0}^{n} u_{i j} P_{i}(x) P_{j}(t)=\Phi^{T}(x) \mathbf{U} \boldsymbol{\Phi}(t),
$$


where

$$
\mathbf{U}=\left[\begin{array}{cccc}
u_{00} & u_{01} & \cdots & u_{0 n} \\
u_{10} & u_{11} & \cdots & u_{1 n} \\
\vdots & \vdots & \ddots & \vdots \\
u_{n 0} & u_{n 1} & \cdots & u_{n n}
\end{array}\right]
$$

Theorem 3 (see [19]). If a continuous function $u(x, t)$, defined on $[0,1] \times[0,1]$, has bounded mixed fourth partial derivative $\partial^{4} u(x, t) / \partial^{2} x \partial t^{2}$, then the Legendre expansion of the function converges uniformly to the function.

For sufficiently smooth function $u(x, t)$ on $[0,1] \times[0,1]$, the error of the approximation is given by

$$
\left\|u(x, t)-P_{\Pi}(x, t)\right\|_{2} \leq\left(C_{1}+C_{2}+C_{3} \frac{1}{\widehat{m}^{\widehat{m}+1}}\right) \frac{1}{\widehat{m}^{\widehat{m}+1}},
$$

where

$$
\begin{aligned}
& C_{1}=\frac{1}{4} \max _{(x, t) \in[0,1] \times[0,1]}\left|\frac{\partial^{\widehat{m}+1} u(x, t)}{\partial x^{\widehat{m}+1}}\right|, \\
& C_{2}=\frac{1}{4} \max _{(x, t) \in[0,1] \times[0,1]}\left|\frac{\partial^{\widehat{m}+1} u(x, t)}{\partial t^{\widehat{m}+1}}\right|, \\
& C_{3}=\frac{1}{16} \max _{(x, t) \in[0,1] \times[0,1]}\left|\frac{\partial^{2 \widehat{m}+2} u(x, t)}{\partial x^{\widehat{m}+1} \partial t^{\widehat{m}+1}}\right| .
\end{aligned}
$$

We refer the reader to [20] for the proof of the above result.

\section{Numerical Solution of the Fractional Partial Differential Equation}

Consider the fractional partial differential equation with variable coefficients equation (1). If we approximate the function $u(x, t)$ with the Legendre polynomials, it can be written as (15). Then we have

$$
\begin{aligned}
& \frac{\partial^{\alpha} u(x, t)}{\partial x^{\alpha}} \simeq \frac{\partial^{\alpha}\left(\Phi^{T}(x) \mathbf{U} \boldsymbol{\Phi}(t)\right)}{\partial x^{\alpha}}=\left(\frac{\partial^{\alpha} \boldsymbol{\Phi}(x)}{\partial x^{\alpha}}\right)^{T} \mathbf{U} \boldsymbol{\Phi}(t)=\left(\frac{\partial^{\alpha} \mathbf{T}_{n}(x)}{\partial x^{\alpha}}\right)^{T} \mathbf{A}^{T} \mathbf{U} \boldsymbol{\Phi}(t) \\
& =\left[0 \frac{\Gamma(2)}{\Gamma(2-\alpha)} x^{1-\alpha} \frac{\Gamma(3)}{\Gamma(3-\alpha)} x^{2-\alpha} \cdots \frac{\Gamma(n+1)}{\Gamma(n+1-\alpha)} x^{n-\alpha}\right] \mathbf{A}^{T} \mathbf{U} \boldsymbol{\Phi}(t)
\end{aligned}
$$

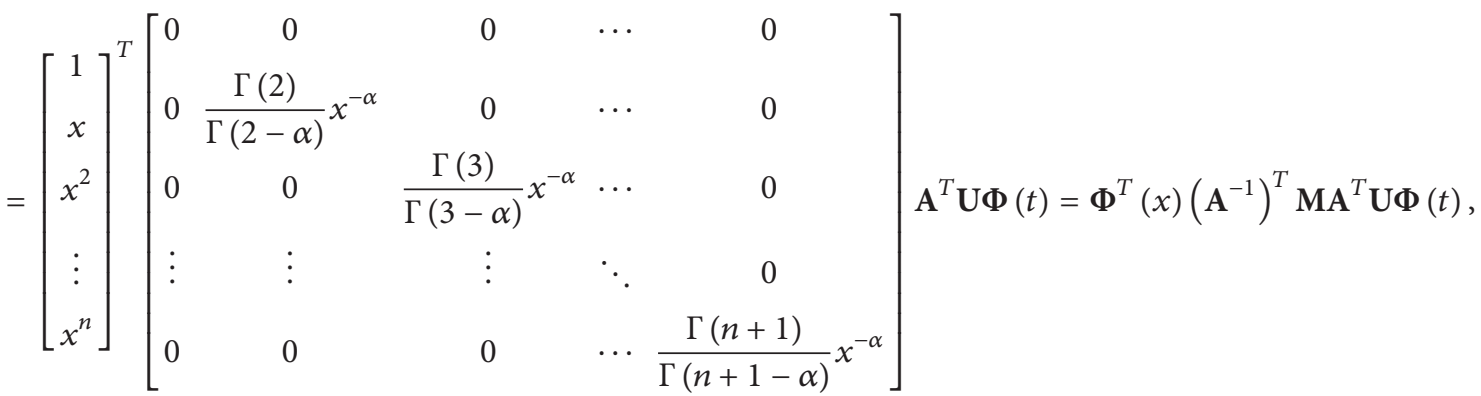

$$
\begin{aligned}
& \frac{\partial^{\beta} u(x, t)}{\partial t^{\beta}} \simeq \frac{\partial^{\beta}\left(\Phi^{T}(x) \mathbf{U} \boldsymbol{\Phi}(t)\right)}{\partial t^{\beta}}=\Phi^{T}(x) \mathbf{U} \frac{\partial^{\beta} \boldsymbol{\Phi}(t)}{\partial t^{\beta}}=\Phi^{T}(x) \mathbf{U A} \frac{\partial^{\beta} \mathbf{T}_{n}(t)}{\partial t^{\beta}} \\
& =\boldsymbol{\Phi}^{T}(x) \mathbf{U A}\left[0 \frac{\Gamma(2)}{\Gamma(2-\beta)} t^{1-\beta} \frac{\Gamma(3)}{\Gamma(3-\beta)} t^{2-\beta} \cdots \frac{\Gamma(n+1)}{\Gamma(n+1-\beta)} t^{n-\beta}\right]^{T} \\
& =\Phi^{T}(x) \mathbf{U A}\left[\begin{array}{ccccc}
0 & 0 & 0 & \cdots & 0 \\
0 & \frac{\Gamma(2)}{\Gamma(2-\beta)} t^{-\beta} & 0 & \cdots & 0 \\
0 & 0 & \frac{\Gamma(3)}{\Gamma(3-\beta)} t^{-\beta} & \cdots & 0 \\
\vdots & \vdots & \vdots & \ddots & 0 \\
0 & 0 & 0 & \cdots & \frac{\Gamma(n+1)}{\Gamma(n+1-\beta)} t^{-\beta}
\end{array}\right] \mathbf{T}_{n}(t)=\boldsymbol{\Phi}^{T}(x) \mathbf{U A N A}^{-1} \boldsymbol{\Phi}(t) .
\end{aligned}
$$


Let

$$
\mathbf{M}=\left[\begin{array}{ccccc}
0 & 0 & 0 & \ldots & 0 \\
0 & \frac{\Gamma(2)}{\Gamma(2-\alpha)} x^{-\alpha} & 0 & \ldots & 0 \\
0 & 0 & \frac{\Gamma(3)}{\Gamma(3-\alpha)} x^{-\alpha} & \ldots & 0 \\
\vdots & \vdots & \vdots & \ddots & 0 \\
0 & 0 & 0 & \cdots & \frac{\Gamma(n+1)}{\Gamma(n+1-\alpha)} x^{-\alpha}
\end{array}\right],
$$

$$
=\left[\begin{array}{ccccc}
0 & 0 & 0 & \cdots & 0 \\
0 & \frac{\Gamma(2)}{\Gamma(2-\beta)} t^{-\beta} & 0 & \cdots & 0 \\
0 & 0 & \frac{\Gamma(3)}{\Gamma(3-\beta)} t^{-\beta} & \ldots & 0 \\
\vdots & \vdots & \vdots & \ddots & 0 \\
0 & 0 & 0 & \cdots & \frac{\Gamma(n+1)}{\Gamma(n+1-\beta)} t^{-\beta}
\end{array}\right] .
$$

Substituting (19) into (1), we have

$$
\begin{aligned}
a(x) & \boldsymbol{\Phi}^{T}(x)\left(\mathbf{A}^{-1}\right)^{T} \mathbf{M A}^{T} \mathbf{U} \boldsymbol{\Phi}(t) \\
& +b(x) \boldsymbol{\Phi}^{T}(x) \mathbf{U A N A}^{-1} \boldsymbol{\Phi}(t)=f(x, t) .
\end{aligned}
$$

Dispersing (21) by the points $\left(x_{i}, t_{j}\right)\left(i=1,2, \ldots, n_{x} ; j=\right.$ $1,2, \ldots, n_{t}$ ), we can obtain $\mathbf{U}$ which is unknown.

\section{Error Analysis}

In this part, in order to illustrate the effectiveness of $\partial^{\alpha} u(x, y) / \partial x^{\alpha} \cong \boldsymbol{\Phi}^{T}(x) \mathbf{U} \boldsymbol{\Phi}(y)$, we have given the following theorem. Let $\partial^{\alpha} u_{n}(x, y) / \partial x^{\alpha}$ be the following approximation of $\partial^{\alpha} u(x, y) / \partial x^{\alpha}$ :

$$
\frac{\partial^{\alpha} u_{n}(x, y)}{\partial x^{\alpha}}=\sum_{i=1}^{n} \sum_{j=1}^{n} u_{i j} P_{i}(x) P_{j}(y) .
$$

Then we have

$$
\frac{\partial^{\alpha} u(x, y)}{\partial x^{\alpha}}-\frac{\partial^{\alpha} u_{n}(x, y)}{\partial x^{\alpha}}=\sum_{i=n+1}^{\infty} \sum_{j=n+1}^{\infty} u_{i j} P_{i}(x) P_{j}(y) .
$$

Theorem 4. Suppose that the function $\partial^{\alpha} u_{n}(x, y) / \partial x^{\alpha}$ obtained by using Legendre polynomials is the approximation of $\partial^{\alpha} u(x, y) / \partial x^{\alpha}$, and $u(x, y)$ has bounded mixed fractional partial derivative $\left|\partial^{4+\alpha+\beta} u(x, y) / \partial x^{2+\alpha} \partial y^{2+\beta}\right| \leq \widehat{M}$; then we have the following upper bound of error:

$$
\left\|\frac{\partial^{\alpha} u(x, y)}{\partial x^{\alpha}}-\frac{\partial^{\alpha} u_{n}(x, y)}{\partial x^{\alpha}}\right\|_{E} \leq \frac{\widehat{M}}{8}\left(\frac{\Gamma^{\prime}(n-0.5)}{\Gamma(n-0.5)}\right)^{\prime \prime \prime},
$$

where $\|u(x, y)\|_{E}=\left(\int_{-1}^{1} \int_{-1}^{1} u^{2}(x, y) d x d y\right)^{1 / 2}$ and

$$
\begin{aligned}
u_{i j}= & \left(\frac{2 i+1}{2}\right)\left(\frac{2 j+1}{2}\right) \\
& \cdot \int_{-1}^{1} \int_{-1}^{1} \frac{\partial^{\alpha} u(x, y)}{\partial x^{\alpha}} P_{i}(x) P_{j}(y) d x d y .
\end{aligned}
$$

Proof. The property of the sequence $\left\{P_{i}(x)\right\}$ on $[-1,1]$ implies that

$$
\int_{-1}^{1} P_{i}(x) P_{j}(x) d x= \begin{cases}\frac{2}{2 i+1}, & i=j \\ 0, & i \neq j\end{cases}
$$

then

$$
\begin{aligned}
& \left\|\frac{\partial^{\alpha} u(x, y)}{\partial x^{\alpha}}-\frac{\partial^{\alpha} u_{n}(x, y)}{\partial x^{\alpha}}\right\|_{E}^{2} \\
& =\int_{-1}^{1} \int_{-1}^{1}\left[\frac{\partial^{\alpha} u(x, y)}{\partial x^{\alpha}}-\frac{\partial^{\alpha} u_{n}(x, y)}{\partial x^{\alpha}}\right]^{2} d x d y \\
& =\int_{-1}^{1} \int_{-1}^{1}\left[\sum_{i=n+1}^{\infty} \sum_{j=n+1}^{\infty} u_{i j} P_{i}(x) P_{j}(y)\right]^{2} d x d y \\
& =\int_{-1}^{1} \int_{-1}^{1} \sum_{i=n+1}^{\infty} \sum_{j=n+1}^{\infty} u_{i j}^{2} P_{i}^{2}(x) P_{j}^{2}(y) d x d y \\
& =\sum_{i=n+1}^{\infty} \sum_{j=n+1}^{\infty} u_{i j}^{2} \int_{-1}^{1} P_{i}^{2}(x) d x \int_{-1}^{1} P_{j}^{2}(y) d y \\
& =\sum_{i=n+1}^{\infty} \sum_{j=n+1}^{\infty} u_{i j}^{2} \frac{2}{2 i+1} \frac{2}{2 j+1} .
\end{aligned}
$$

The Legendre polynomials coefficients of function $\partial^{\alpha} u(x, y)$ / $\partial x^{\alpha}$ are given by

$$
\begin{aligned}
u_{i j}= & \left(\frac{2 i+1}{2}\right)\left(\frac{2 j+1}{2}\right) \\
& \cdot \int_{-1}^{1} \int_{-1}^{1} \frac{\partial^{\alpha} u(x, y)}{\partial x^{\alpha}} P_{i}(x) P_{j}(y) d x d y .
\end{aligned}
$$

Therefore, we obtain

$$
\begin{aligned}
u_{i j} & =\frac{2 j+1}{4} \int_{-1}^{1} \frac{\partial^{\alpha} u(x, y)}{\partial x^{\alpha}}\left[P_{i+1}(x)-P_{i-1}(x)\right] \\
\cdot & \left.P_{j}(y)\right|_{-1} ^{1} d y-\frac{2 j+1}{4} \\
& \cdot \int_{-1}^{1} \int_{-1}^{1} \frac{\partial^{\alpha+1} u(x, y)}{\partial x^{\alpha+1}}\left[P_{i+1}(x)-P_{i-1}(x)\right] P_{j}(y) d x d y \\
& =-\frac{2 j+1}{4} \int_{-1}^{1} \int_{-1}^{1} \frac{\partial^{\alpha+1} u(x, y)}{\partial x^{\alpha+1}}\left[P_{i+1}(x)\right. \\
& \left.-P_{i-1}(x)\right] P_{j}(y) d x d y=-\frac{2 j+1}{4}
\end{aligned}
$$




$$
\begin{aligned}
& \int_{-1}^{1} \frac{\partial^{\alpha+1} u(x, y)}{\partial x^{\alpha+1}}\left[\frac{P_{i+2}(x)-P_{i}(x)}{2 i+3}\right. \\
& \left.-\frac{P_{i}(x)-P_{i-2}(x)}{2 i-1}\right]\left.P_{j}(y)\right|_{-1} ^{1} d y+\frac{2 j+1}{4} \\
& \cdot \int_{-1}^{1} \int_{-1}^{1} \frac{\partial^{\alpha+2} u(x, y)}{\partial x^{\alpha+2}}\left[\frac{P_{i+2}(x)-P_{i}(x)}{2 i+3}\right. \\
& \left.-\frac{P_{i}(x)-P_{i-2}(x)}{2 i-1}\right] P_{j}(y) d x d y=\frac{2 j+1}{4} \\
& \cdot \int_{-1}^{1} \int_{-1}^{1} \frac{\partial^{\alpha+2} u(x, y)}{\partial x^{\alpha+2}}\left[\frac{P_{i+2}(x)-P_{i}(x)}{2 i+3}\right. \\
& \left.-\frac{P_{i}(x)-P_{i-2}(x)}{2 i-1}\right] P_{j}(y) d x d y .
\end{aligned}
$$

Now, let $\tau_{i}(x)=(2 i-1) P_{i+2}(x)-2(2 i+1) P_{i}(x)+(2 i+3) P_{i-2}(x)$; then we have

$$
\begin{aligned}
u_{i j}= & \frac{2 j+1}{4(2 i-1)(2 i+3)} \\
& \cdot \int_{-1}^{1} \int_{-1}^{1} \frac{\partial^{2+\alpha} u(x, y)}{\partial x^{2+\alpha}} \tau_{i}(x) P_{j}(y) d x d y .
\end{aligned}
$$

By solving this equation, we have

$$
\begin{aligned}
u_{i j}= & \frac{1}{4(2 i-1)(2 i+3)(2 j-1)(2 j+3)} \\
& \cdot \int_{-1}^{1} \int_{-1}^{1} \frac{\partial^{4+\alpha+\beta} u(x, y)}{\partial x^{2+\alpha} \partial y^{2+\beta}} \tau_{i}(x) \tau_{j}(y) d x d y .
\end{aligned}
$$

So we have

$$
\begin{aligned}
& \left|u_{i j}\right| \leq \frac{1}{4(2 i-1)(2 i+3)(2 j-1)(2 j+3)} \\
& \cdot \int_{-1}^{1} \int_{-1}^{1}\left|\frac{\partial^{4+\alpha+\beta} u(x, y)}{\partial x^{2+\alpha} \partial y^{2+\beta}}\right|\left|\tau_{i}(x)\right|\left|\tau_{j}(y)\right| d x d y \\
& \leq \frac{\widehat{M}}{4(2 i-1)(2 i+3)(2 j-1)(2 j+3)} \\
& \cdot \int_{-1}^{1}\left|\tau_{i}(x)\right| d x \int_{-1}^{1}\left|\tau_{j}(y)\right| d y .
\end{aligned}
$$

Moreover, it was easily obtained that

$$
\int_{-1}^{1}\left|\tau_{m}(t)\right| d t \leq \sqrt{24} \frac{2 i+3}{\sqrt{2 i-3}}
$$

thus, we have

$$
\begin{gathered}
\left|u_{i j}\right| \leq \frac{24 \widehat{M}}{4(2 i-1)(2 i+3)(2 j-1)(2 j+3)} \frac{(2 i+3)}{\sqrt{2 i-3}} \\
\cdot \frac{(2 j+3)}{\sqrt{2 j-3}} \leq \frac{6 \widehat{M}}{(2 i-3)^{3 / 2}(2 j-3)^{3 / 2}} .
\end{gathered}
$$

Namely

$$
\left|u_{i j}\right|^{2} \leq \frac{36 \widehat{M}^{2}}{(2 i-3)^{3}(2 j-3)^{3}} .
$$

Therefore, we have

$$
\begin{aligned}
& \left\|\frac{\partial^{\alpha} u(x, y)}{\partial x^{\alpha}}-\frac{\partial^{\alpha} u_{n}(x, y)}{\partial x^{\alpha}}\right\|_{E}^{2} \\
& \leq \sum_{i=n+1}^{\infty} \sum_{j=n+1}^{\infty} u_{i j}^{2} \frac{2}{2 i+1} \frac{2}{2 j+1} \\
& \leq \sum_{i=n+1}^{\infty} \sum_{j=n+1}^{\infty} \frac{144 \widehat{M}^{2}}{(2 i-3)^{3}(2 j-3)^{3}(2 i+1)(2 j+1)} \\
& \leq \sum_{i=n+1}^{\infty} \sum_{j=n+1}^{\infty} \frac{144 \widehat{M}^{2}}{(2 i-3)^{4}(2 j-3)^{4}} \\
& \quad=\left[\sum_{i=n+1}^{\infty} \frac{12 \widehat{M}}{(2 i-3)^{4}}\right]^{2}=\left[\frac{\widehat{M}}{8}\left(\frac{\Gamma^{\prime}(n-0.5)}{\Gamma(n-0.5)}\right)^{\prime \prime \prime}\right]^{2}
\end{aligned}
$$

thus

$$
\left\|\frac{\partial^{\alpha} u(x, y)}{\partial x^{\alpha}}-\frac{\partial^{\alpha} u_{n}(x, y)}{\partial x^{\alpha}}\right\|_{E} \leq \frac{\widehat{M}}{8}\left(\frac{\Gamma^{\prime}(n-0.5)}{\Gamma(n-0.5)}\right)^{\prime \prime \prime} .
$$

This completes the proof.

\section{Numerical Examples}

Example 1. Consider the following nonhomogeneous partial differential equation:

$$
\begin{aligned}
& x^{1 / 3} \frac{\partial^{1 / 2} u(x, t)}{\partial x^{1 / 2}}+x^{1 / 2} \frac{\partial^{1 / 2} u(x, t)}{\partial t^{1 / 2}}=f(x, t) \\
& (x, t) \in[0,1] \times[0,1], u(x, 0)=10 x^{2}(1-x), u(0, t)=u(1, t)=0,
\end{aligned}
$$

where $f(x, t)=-40 \sqrt{t}(3+2 t)(-1+x) x^{7 / 3} / 3 \sqrt{\pi}-16(1+$ $t)^{2} x^{2}(-5+6 x) / 3 \sqrt{\pi}$. The exact solution of this equation is $u(x, t)=10 x^{2}(1-x)(1+t)^{2}$. Tables $1-3$ show the absolute errors for $t=1 / 4 s, t=1 / 2 s$, and $t=3 / 4 s$ of different $n$. 
TABLE 1: Absolute error for $t=1 / 4 \mathrm{~s}$ and different values of $n$.

\begin{tabular}{lccc}
\hline$x$ & $n=2$ & $n=3$ & $n=4$ \\
\hline 0.1 & 0.3246 & $3.2831 e-015$ & $3.3582 e-016$ \\
0.2 & 0.2351 & $4.2734 e-015$ & $5.2745 e-015$ \\
0.3 & 0.4820 & $4.1237 e-015$ & $5.6521 e-015$ \\
0.4 & 0.3274 & $5.7320 e-015$ & $6.2742 e-016$ \\
0.5 & 0.8231 & $4.7381 e-015$ & $7.1640 e-016$ \\
0.6 & 0.9127 & $7.3722 e-015$ & $3.2356 e-016$ \\
0.7 & 1.2188 & $6.3276 e-015$ & $4.2745 e-015$ \\
0.8 & 1.5181 & $1.2374 e-014$ & $3.7224 e-015$ \\
0.9 & 0.8364 & $3.1744 e-015$ & $8.8874 e-015$ \\
\hline
\end{tabular}

TABLE 2: Absolute error for $t=1 / 2 \mathrm{~s}$ and different values of $n$.

\begin{tabular}{lccc}
\hline$x$ & $n=2$ & $n=3$ & $n=4$ \\
\hline 0.1 & 0.2821 & $4.2137 e-015$ & $3.4325 e-016$ \\
0.2 & 0.4375 & $5.8711 e-015$ & $6.5332 e-016$ \\
0.3 & 0.1021 & $4.2210 e-015$ & $5.2435 e-016$ \\
0.4 & 0.2387 & $3.1016 e-016$ & $4.9722 e-016$ \\
0.5 & 0.8277 & $3.4762 e-016$ & $5.3478 e-015$ \\
0.6 & 0.9322 & $5.3265 e-015$ & $1.3241 e-016$ \\
0.7 & 1.3846 & $4.7632 e-015$ & $9.2371 e-016$ \\
0.8 & 1.6654 & $4.2346 e-016$ & $8.4812 e-015$ \\
0.9 & 0.9144 & $2.0437 e-016$ & $6.3273 e-016$ \\
\hline
\end{tabular}

TABle 3: Absolute error for $t=3 / 4 \mathrm{~s}$ and different values of $n$.

\begin{tabular}{lccc}
\hline$x$ & $n=2$ & $n=3$ & $n=4$ \\
\hline 0.1 & 0.3126 & $5.0832 e-016$ & $5.3281 e-016$ \\
0.2 & 0.8978 & $4.1845 e-016$ & $2.3258 e-015$ \\
0.3 & 0.2374 & $2.3448 e-016$ & $4.0112 e-016$ \\
0.4 & 0.2951 & $3.2155 e-016$ & $5.1223 e-016$ \\
0.5 & 0.3327 & $5.5518 e-015$ & $6.3274 e-015$ \\
0.6 & 1.3267 & $6.2440 e-016$ & $5.7421 e-015$ \\
0.7 & 0.8723 & $3.5220 e-016$ & $5.2871 e-016$ \\
0.8 & 0.9229 & $4.2301 e-016$ & $4.8810 e-016$ \\
0.9 & 1.1327 & $5.2310 e-015$ & $6.1138 e-016$ \\
\hline
\end{tabular}

From Tables 1-3, we can see that the absolute error is very small when $n \geq 3$. Also, when $n$ is fixed, the more points we take, the more accurate numerical solutions we obtain. Figures 1-3 show the fact that $n_{x, t}$ is the number of $x_{i}, t_{j}$.

Example 2. Consider the following fractional partial differential equation:

$$
\begin{aligned}
& x \frac{\partial^{1 / 4} u(x, t)}{\partial x^{1 / 4}}+x^{2 / 3} \frac{\partial^{1 / 3} u(x, t)}{\partial t^{1 / 3}}=f(x, t), \\
& u(x, 0)=x(x-1)\left(x^{2}+1\right), u(0, t)=0,
\end{aligned}
$$

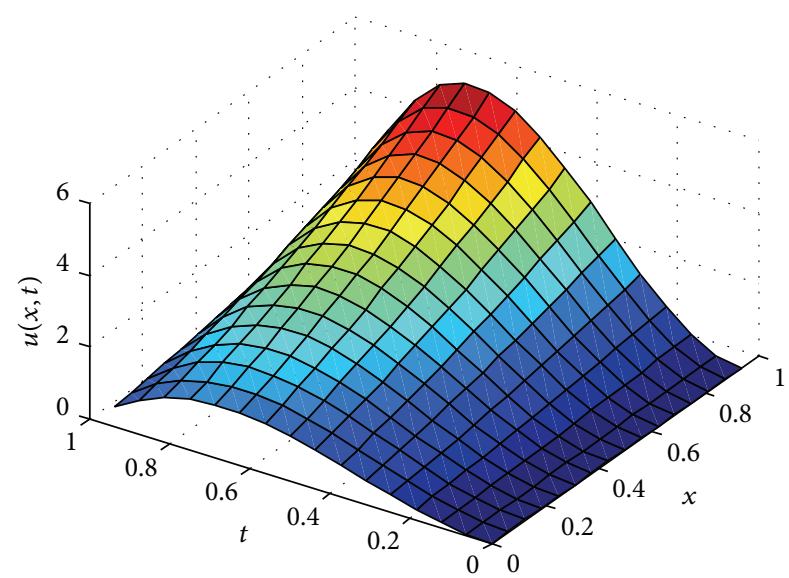

FIgURE 1: Numerical solution of $n_{x, t}=3$.

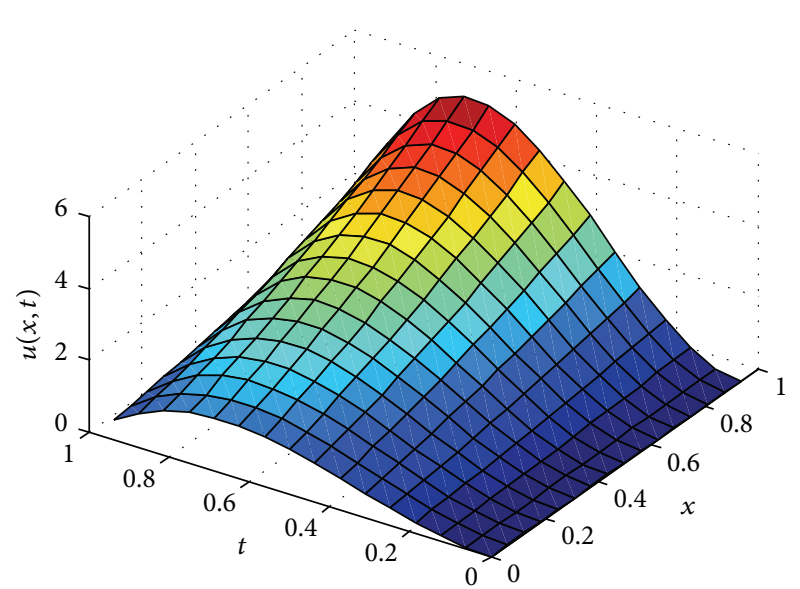

FIGURE 2: Numerical solution of $n_{x, t}=6$.

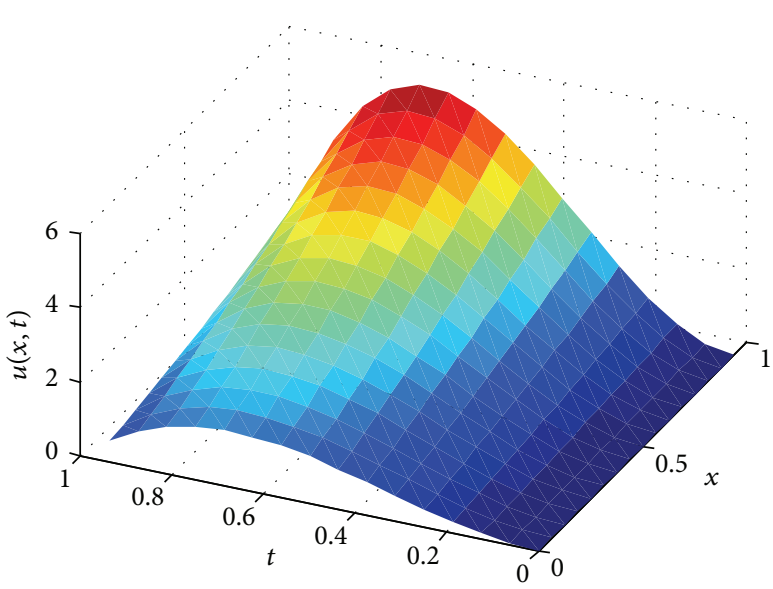

Figure 3: Exact solution.

where

$$
\begin{aligned}
& f(x, t)=\frac{3 t^{2 / 3}\left(55+66 t+81 t^{3}\right)(-1+x) x^{2}\left(1+x^{2}\right)}{110 \Gamma(2 / 3)} \\
& +\frac{4\left(1+t+t^{2}+t^{4}\right) x^{17 / 12}\left[-385+8 x\left(55-60 x+64 x^{2}\right)\right]}{1155 \Gamma(3 / 4)} .
\end{aligned}
$$




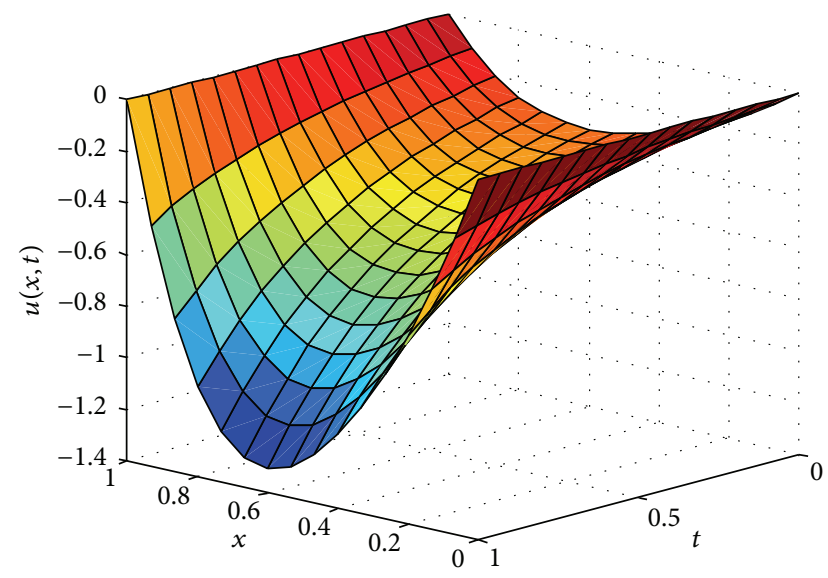

Figure 4: The numerical solutions for Example 2 when $n=4$.

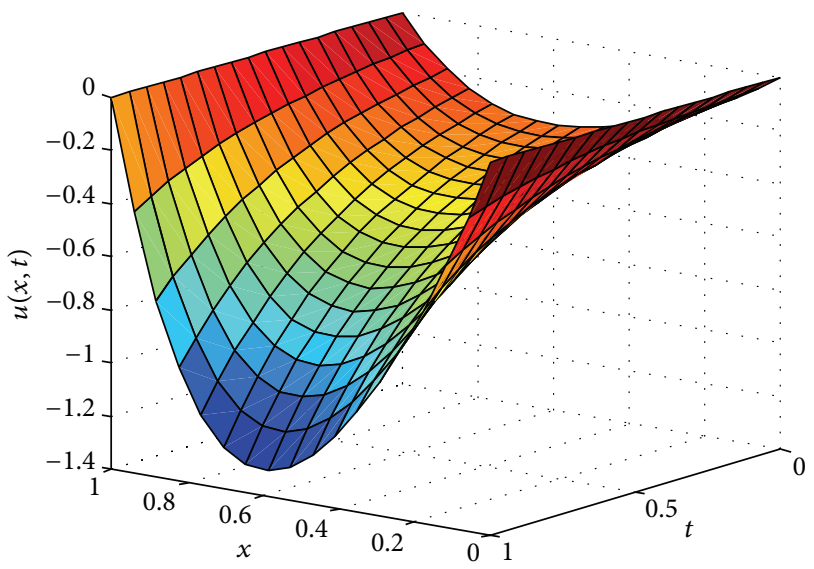

Figure 5: The numerical solutions for Example 2 when $n=5$.

The exact solution is $u(x, t)=x(x-1)\left(x^{2}+1\right)\left(1+t+t^{2}+t^{4}\right)$. The numerical solutions for $n=4, n=5$ are displayed in Figures 4 and 5 and the exact solution is shown in Figure 6.

Example 3. Consider this equation:

$$
\begin{aligned}
& x^{1 / 2} \frac{\partial^{1 / 3} u(x, t)}{\partial x^{1 / 3}}+x^{2 / 3} \frac{\partial^{1 / 3} u(x, t)}{\partial t^{1 / 3}}=f(x, t), \\
& u(x, 0)=x^{2}-1, u(0, t)=-1-(t-1)^{2}-(t-1)^{3},
\end{aligned}
$$

where

$$
\begin{aligned}
f(x, t) & \\
= & \frac{9\left(1+t-2 t^{2}+t^{3}\right) x^{13 / 6}}{5 \Gamma(2 / 3)} \\
& +\frac{3 t^{2 / 3}(-2+3 t)(-10+9 t)\left(-1+x^{2}\right) x^{2 / 3}}{40 \Gamma(2 / 3)} .
\end{aligned}
$$

The exact solution is $u(x, t)=\left(x^{2}-1\right)\left[1+(t-1)^{2}+(t-1)^{3}\right]$. The numerical solutions for $n=3, n=4$ are displayed in Figures 7 and 8 . The absolute errors are shown in Figures 9 and 10 when $n=3$ and $n=4$.

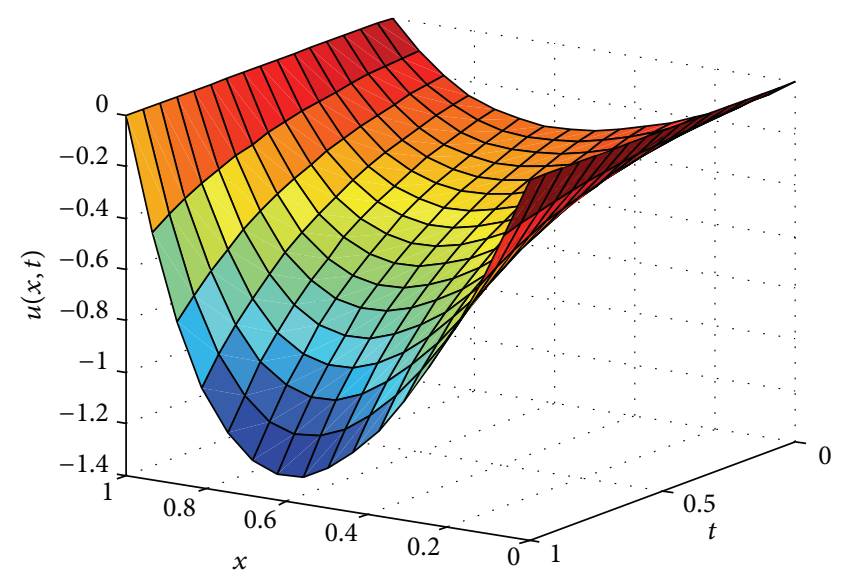

FIGURE 6: Exact solution for Example 2.

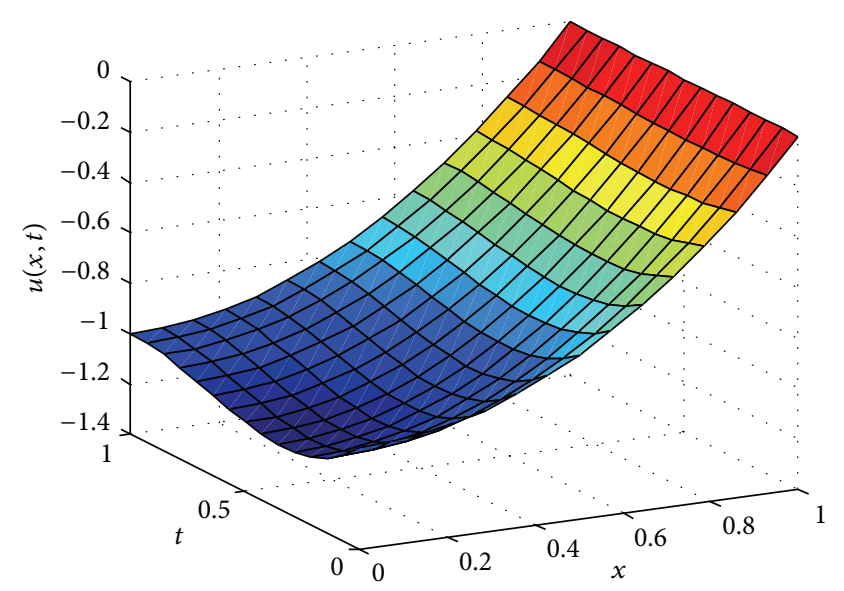

FIgURE 7: The numerical solutions for Example 3 when $n=3$.

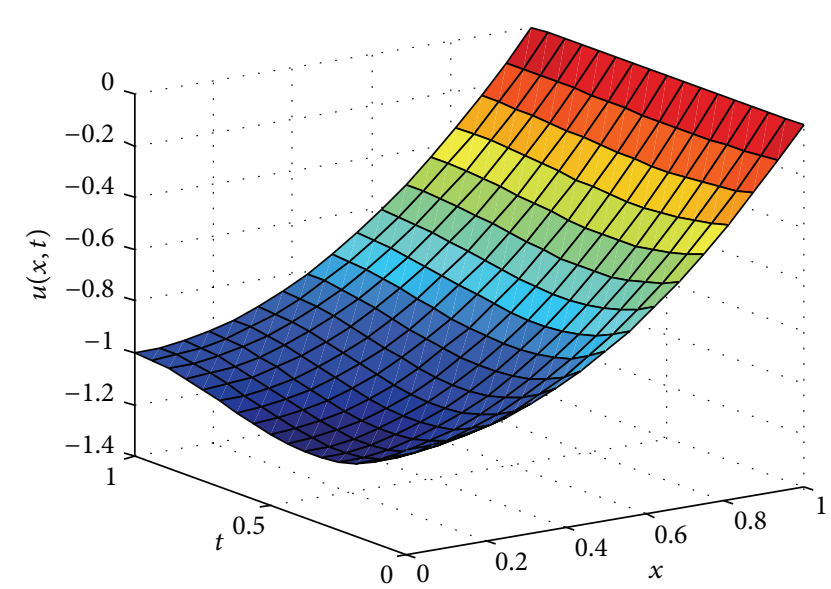

FIgURE 8: The numerical solutions for Example 3 when $n=4$.

From Examples 1-3, we can see that the method in this paper can be effectively used to solve the numerical solution of fractional partial differential equation with variable coefficients. From the above results, the absolute errors between 


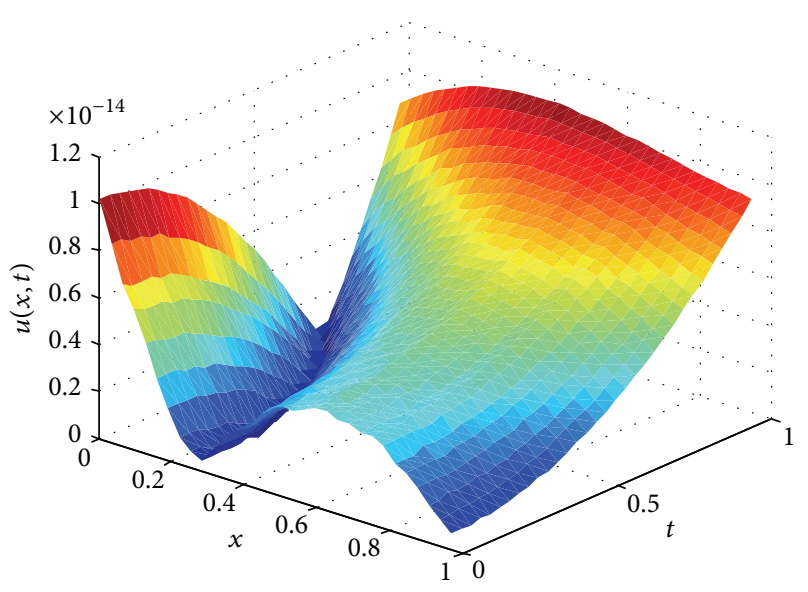

Figure 9: The absolute errors for Example 3 when $n=3$.

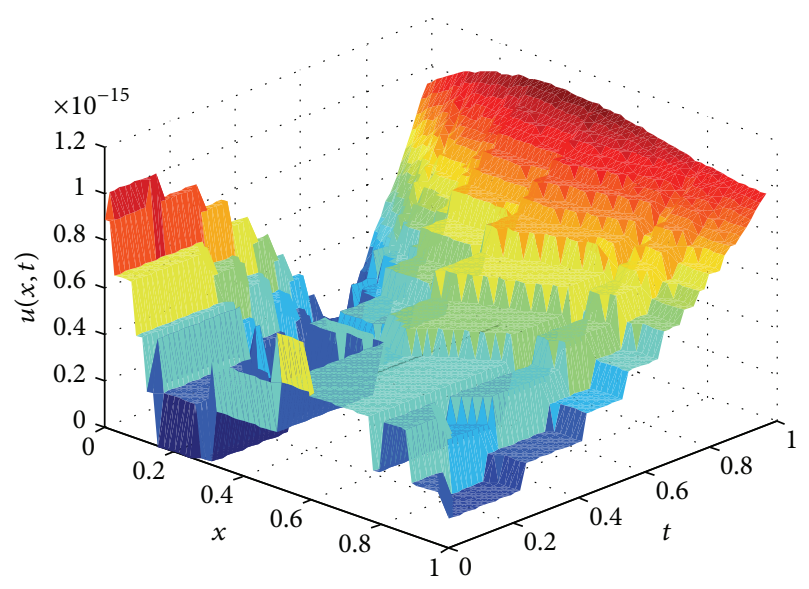

Figure 10: The absolute errors for Example 3 when $n=4$.

the numerical solutions and the exact solution are rather small. What is more, due to the absolute error in this paper is about $10^{-15}$, the Legendre polynomials method can reach higher degree of accuracy by comparing the approximations obtained by block pulse method [16].

\section{Conclusion}

In this paper, we use the Legendre polynomials method to solve a class of fractional partial differential equations with variable coefficients. The Legendre polynomials operational matrix of fractional differentiation is derived from the property of Legendre polynomials. The initial equation is translated into the product of some relevant matrixes, which can also be regarded as the system of linear equations. The error analysis of Legendre polynomials is also given. The numerical results show that numerical solutions obtained by our method are in very good agreement with the exact solution.

\section{Conflict of Interests}

The authors declare that there is no conflict of interests regarding the publication of this paper.

\section{References}

[1] R. L. Bagley and R. A. Calico, "Fractional order state equations for the control of viscoelastically damped structures," Journal of Guidance, Control, and Dynamics, vol. 14, no. 2, pp. 304-311, 1991.

[2] Z. C. Li and J. S. Luo, Wavelet Analysis and Its Application, Electronic Industrial Publication, Beijing, China, 2005.

[3] J. H. Chen, "Analysis of stability and convergence of numerical approximation for the Riesz fractional reaction-dispersion equation," Journal of Xiamen University (Natural Science), vol. 46, no. 5, pp. 616-619, 2007.

[4] D. Delbosco and L. Rodino, "Existence and uniqueness for a nonlinear fractional differential equation," Journal of Mathematical Analysis and Applications, vol. 204, no. 2, pp. 609-625, 1996.

[5] Y. Ryabov and A. Puzenko, "A damped oscillations in view of the fraction oscillator equation," Physics Review B, vol. 66, pp. 184-201, 2002.

[6] A. M. El-Sayed, "Nonlinear functional-differential equations of arbitrary orders," Nonlinear Analysis: Theory, Methods \& Applications, vol. 33, no. 2, pp. 181-186, 1998.

[7] I. L. El-Kalla, "Error estimate of the series solution to a class of nonlinear fractional differential equations," Communications in Nonlinear Science and Numerical Simulation, vol. 16, no. 3, pp. 1408-1413, 2011.

[8] Z. M. Odibat, "A study on the convergence of variational iteration method," Mathematical and Computer Modelling, vol. 51, no. 9-10, pp. 1181-1192, 2010.

[9] S. Momani, Z. Odibat, and V.S. Erturk, "Generalized differential transform method for solving a space- and time-fractional diffusion-wave equation," Physics Letters A, vol. 370, no. 5-6, pp. 379-387, 2007.

[10] Z. Odibat, S. Momani, and V. S. Erturk, "Generalized differential transform method: application to differential equations of fractional order," Applied Mathematics and Computation, vol. 197, no. 2, pp. 467-477, 2008.

[11] Z. Odibat and S. Momani, "A generalized differential transform method for linear partial differential equations of fractional order," Applied Mathematics Letters, vol. 21, no. 2, pp. 194-199, 2008.

[12] Y. Zhang, "A finite difference method for fractional partial differential equation," Applied Mathematics and Computation, vol. 215, no. 2, pp. 524-529, 2009.

[13] Y. X. Wang and Q. B. Fan, "The second kind Chebyshev wavelet method for solving fractional differential equations," Applied Mathematics and Computation, vol. 218, no. 17, pp. 8592-8601, 2012.

[14] M. X. Yi and Y. M. Chen, "Haar wavelet operational matrix method for solving fractional partial differential equations," Computer Modeling in Engineering \& Sciences, vol. 88, no. 3, pp. 229-244, 2012.

[15] E. H. Doha, A. H. Bhrawy, D. Baleanu, and S. S. Ezz-Eldien, “The operational matrix formulation of the Jacobi tau approximation for space fractional diffusion equation," Advances in Difference Equations, vol. 231, pp. 1687-1847, 2014.

[16] M. X. Yi, J. Huang, and J. X. Wei, "Block pulse operational matrix method for solving fractional partial differential equation," Applied Mathematics and Computation, vol. 221, pp. 121131, 2013. 
[17] I. Podlubny, Fractional Differential Equations, vol. 198 of Mathematics in Science and Engineering, Academic Press, New York, NY, USA, 1999.

[18] A. Saadatmandi and M. Dehghan, "A new operational matrix for solving fractional-order differential equations," Computers and Mathematics with Applications, vol. 59, no. 3, pp. 1326-1336, 2010.

[19] N. Liu and E.-B. Lin, "Legendre wavelet method for numerical solutions of partial differential equations," Numerical Methods for Partial Differential Equations, vol. 26, no. 1, pp. 81-94, 2010.

[20] S. Nemati and Y. Ordokhani, "Legendre expansion methods for the numerical solution of nonlinear 2D Fredholm integral equations of the second kind," Journal of Applied Mathematics \& Informatics, vol. 31, no. 5-6, pp. 609-621, 2013. 


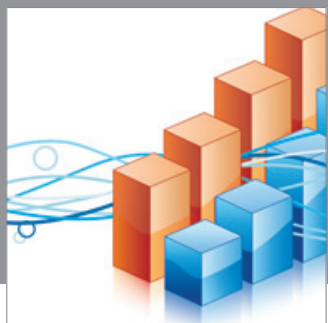

Advances in

Operations Research

mansans

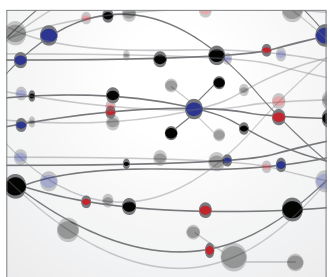

The Scientific World Journal
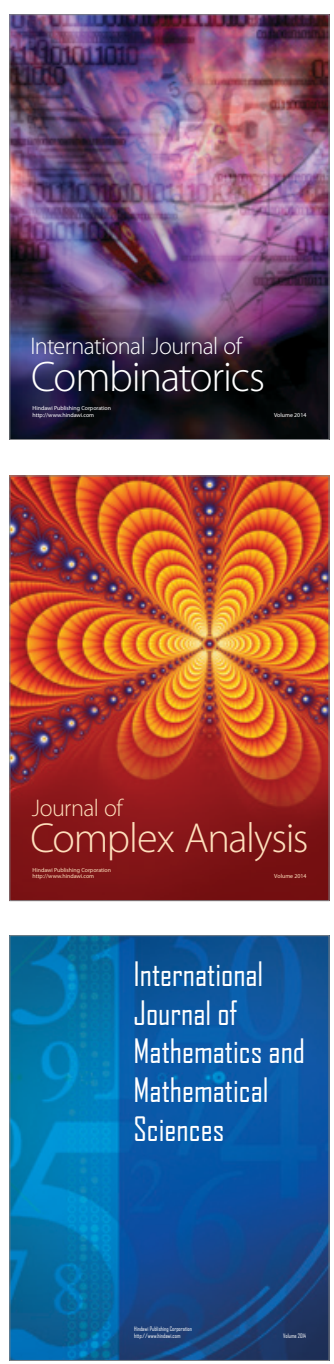
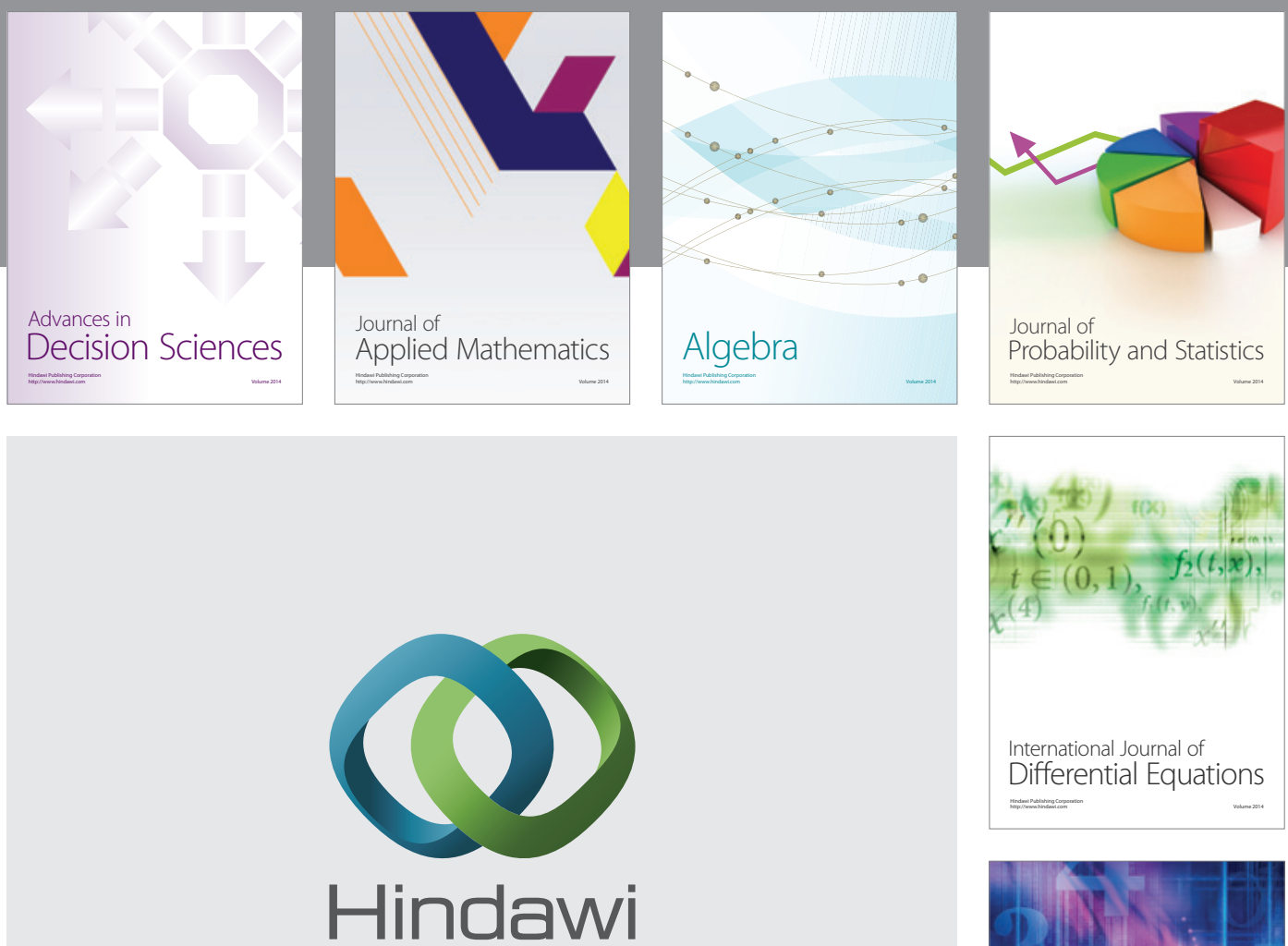

Submit your manuscripts at http://www.hindawi.com
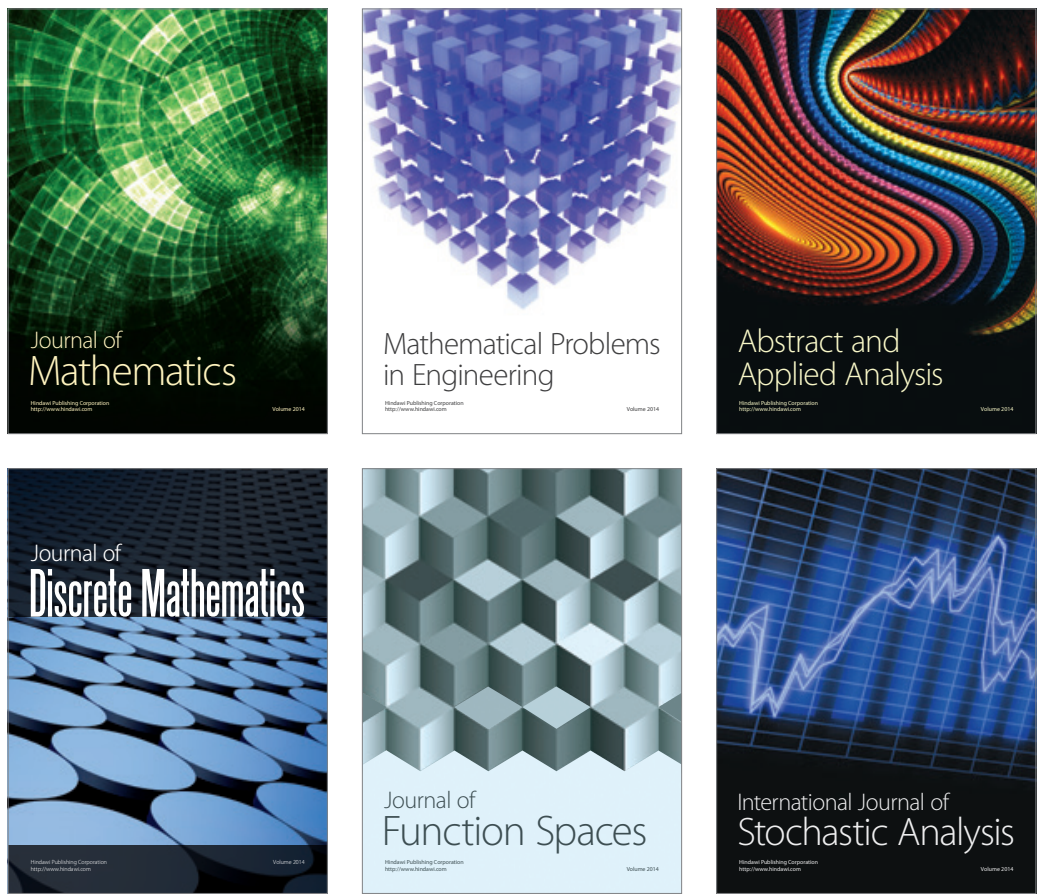

Journal of

Function Spaces

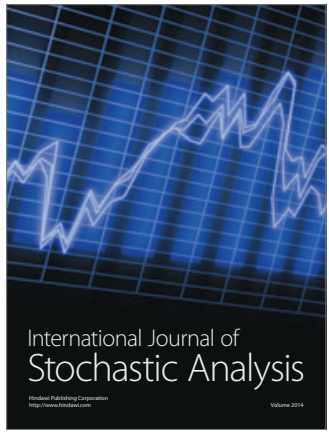

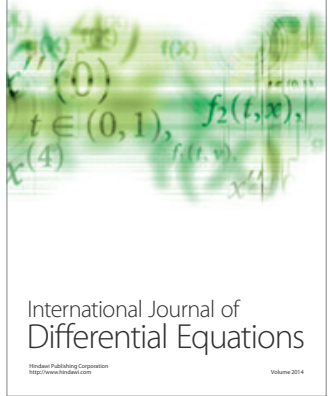
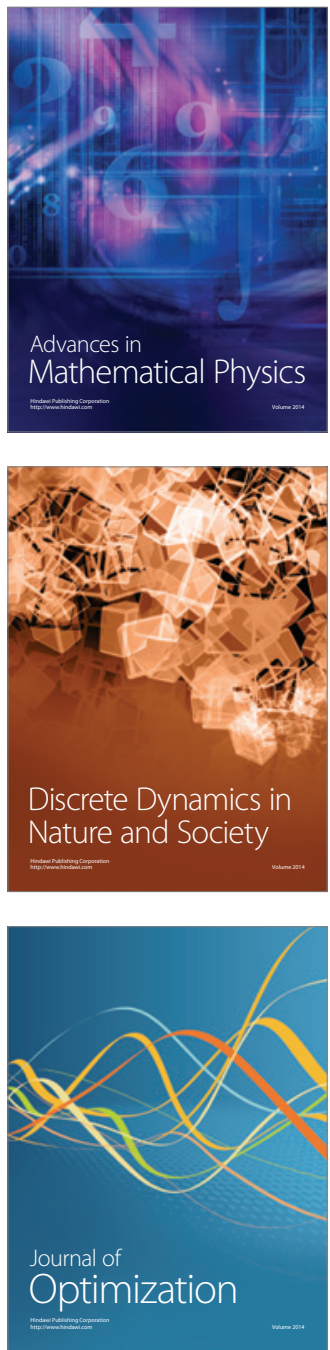